\title{
The identification of SS73 71 as a new symbiotic star ${ }^{\star}$
}

\author{
C. B. Pereira, C. S. Franco, and S. J. C. Landaberry \\ Observatório Nacional-MCT, Rua José Cristino, 77, CEP 20921-400, São Cristóvão, Rio de Janeiro-RJ, Brazil
}

Received 3 December 2001 / Accepted 17 January 2002

\begin{abstract}
We report spectroscopic observations of a new symbiotic star. This star is located in our Galaxy in the direction of the bulge and was discovered during a southern spectroscopic survey of $\mathrm{H} \alpha$ emission line objects. The star, SS73 71, after Sanduleak \& Stephenson (1973), shows characteristics of a symbiotic star: the presence of TiO bands and emission lines of ions of higher ionization such as He II4686 $\AA$ and [O III] $5007 \AA$. Here, we present some of the main spectroscopic features, line intensities and, whenever possible, some physical parameters.
\end{abstract}

Key words. binaries: symbiotic - stars: emission line, Be

\section{Introduction}

As part of a long-term program to investigate emissionline objects in the southern hemisphere, we recently started a spectroscopic survey at the European Southern Observatory (ESO) of some stars from the Sanduleak \& Stephenson (1973) sample. This survey is also part of a program to study emission-line objects whose nature is not well established in the literature. A previous study of one star from the SS73 list led to the analysis of a peculiar emission-line object, SS73 11 (Landaberry et al. 2001).

In this work we present spectroscopic observations of the emission-line star SS7371, first recognized as an $\mathrm{H} \alpha$ emission line object after a objective prism survey of Sanduleak \& Stephenson (1973), hereafter SS73. SS73 classified this object as a "Very-Steep-BalmerDecrement", $X$ symbol in their Col. 7, which might be an indication of symbiotic object or a highly reddened Be star. Allen (1978) classified it as a "Be star with weak Balmer emission and He I emission". We find that SS73 71 displays characteristics of a symbiotic object, e.g., TiO absorption bands and emission lines of ions of higher ionization such as He II4686 $\AA$ and [O III] $5007 \AA$. This symbiotic is not given in the recent catalogue of Belczyński et al. (2000). A finding chart of the object is present in Fig. 1. The equatorial coordinates of SS7371 are $\alpha(2000.0)=$ $16^{\mathrm{h}} 59^{\mathrm{m}} 23.4^{\mathrm{s}}$ and $\delta(2000.0)=-32^{\circ} 14^{\prime} 43^{\prime \prime}$. The $V$ magnitude was derived convolving the calibrated spectrum with a $V$ filter profile. The result is shown in Table 2 .

Send offprint requests to: C. B. Pereira, e-mail: claudio@on.br

* Based on observations made with the $1.52 \mathrm{~m}$ telescope at the European Southern Observatory (La Silla, Chile) under the agreement with the Observatório Nacional, Brazil.

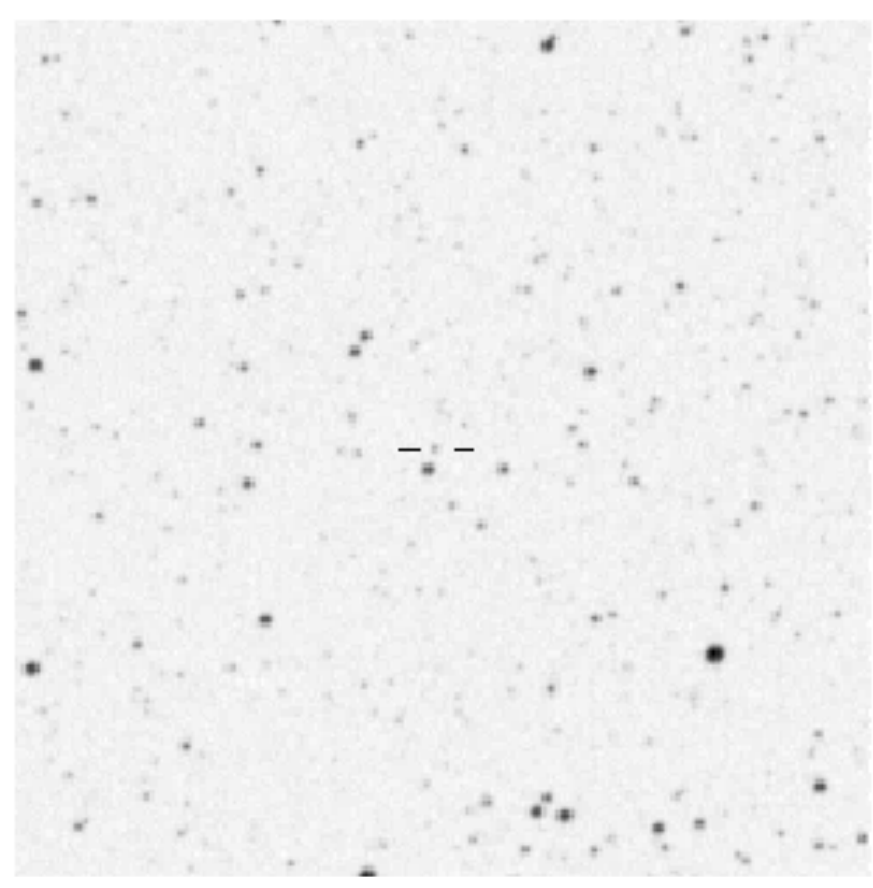

Fig. 1. Finding chart of the region around SS7371. SS73 71 is the faint star at the center of the field. The field has dimensions of $7^{\prime} \times 7^{\prime}$. North is at the top and east is to the left.

\section{Observations and reduction}

Spectroscopic observations were performed using a Boller \& Chivens spectrograph at the Cassegrain focus of the ESO $1.52 \mathrm{~m}$ telescope in La Silla (Chile) at March 24, 2000, June 15, 2001 and September 25, 2001. A UVflooded thinned Loral Lesser CCD \#39 (2048 × 2048, $15 \mu \mathrm{m} /$ pixel) was used as the detector; it gives a high quantum efficiency in the blue and in the UV range. 


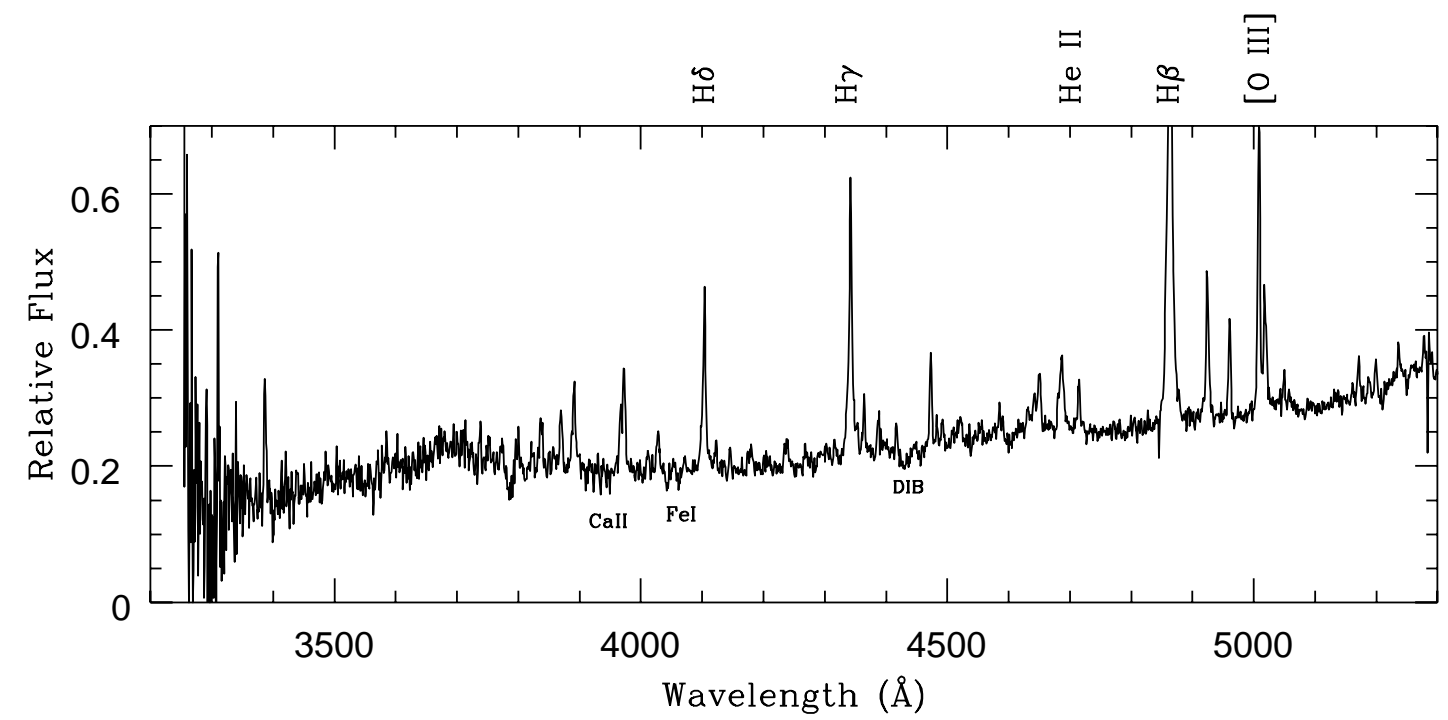

Fig. 2. Blue spectrum of SS7371. Notice the presence of the He II4686 $\AA$ line and the [O III] forbidden line at $5007 \AA$.

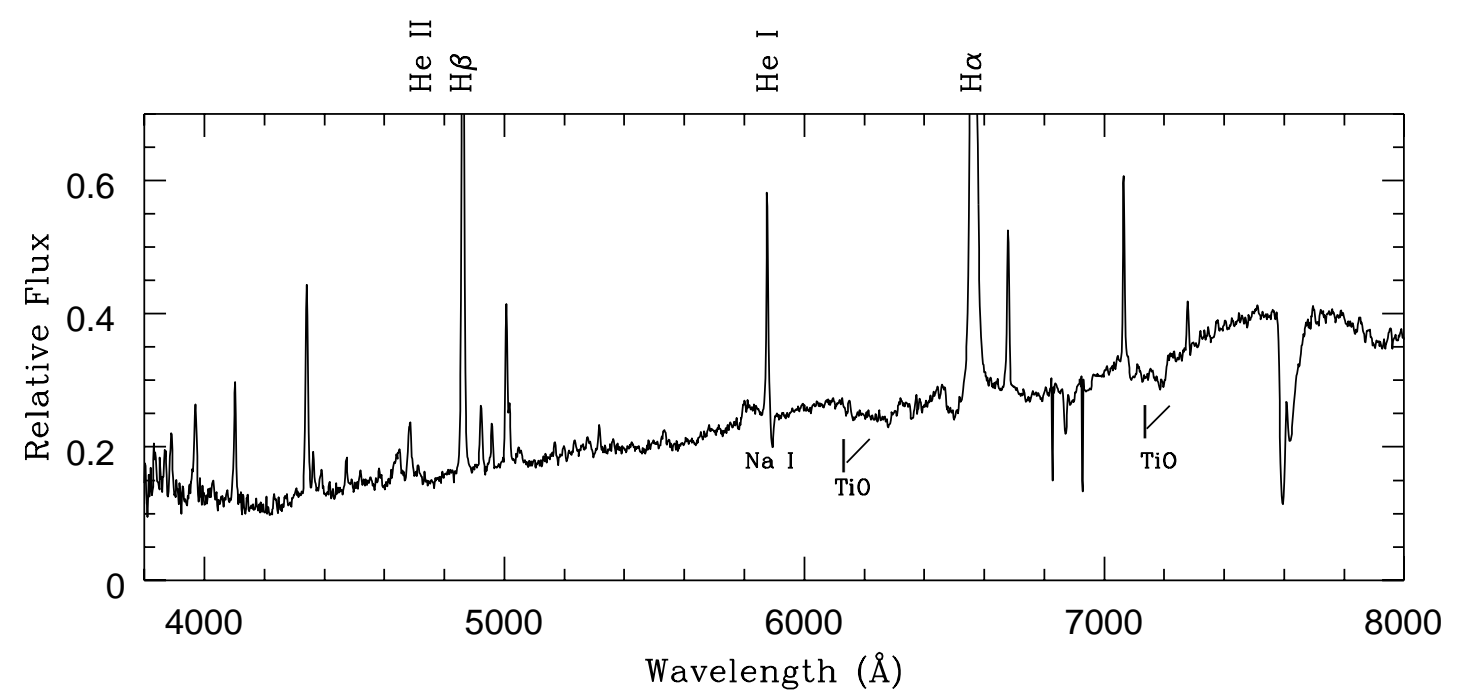

Fig. 3. Optical spectrum of SS73 71.

The setup employed is the same as that used to investigate suspected symbiotic objects with the $1.52 \mathrm{~m}$ telescope (Pereira et al. 1998). Two different instrumental setups were employed. The first one made use of the grating \#23 with $600 \mathrm{l} / \mathrm{mm}$ providing a resolution of $4.6 \AA$ in the range $\lambda \lambda 3500 \AA-8000 \AA$ and the other was the grating \#33 with $1200 \mathrm{l} / \mathrm{mm}$, resulting in a resolution of $1.9 \AA$ in the $\lambda \lambda 3200 \AA-5300 \AA$ range.

The spectra were reduced using standard IRAF tasks, from bias subtraction and flat-field correction, through spectral extraction and wavelength and flux calibration. Spectrophotometric standards from Oke (1974) and Hamuy et al. (1994) were also observed. The slit orientation in the range $(3200 \AA-5300 \AA)$ was aligned with the parallactic angle in order to minimize the light loss due to atmospheric refraction.
The flux of emission lines have been measured by the conventional method adjusting a Gaussian function to the line profile thereby obtaining the intensity, the central wavelength and the line width at half power level. Uncertainties in the line intensities come mainly from the position of the underlying continuum. We estimate the flux error to be about $20 \%$ for the weak lines (line fluxes $\approx 10$ on the scale of $\mathrm{H} \beta=100$ ) and about $10 \%$ for stronger lines. Figures 2 and 3 show the reduced spectra.

\section{The spectrum of SS7371}

Table 1 shows the observed line flux of the identified lines. The spectrum of SS73 71 shows typical features of a symbiotic star with late K-early M component when compared with spectral atlas at same resolution (Jacoby et al. 1984; 
Table 1. Observed emission line fluxes relative to $\mathrm{H} \beta=100$.

\begin{tabular}{|c|c|c|}
\hline Wavelength & Identification & $F(\lambda)$ \\
\hline 3869 & [Ne III] & 6.6 \\
\hline \multirow[t]{2}{*}{3889} & He I & 9.4 \\
\hline & $\mathrm{H} 8$ & \\
\hline \multirow[t]{2}{*}{3968} & [Ne III] & 18.1 \\
\hline & $\mathrm{H} \epsilon$ & \\
\hline 4009 & He I & 3.4 \\
\hline 4026 & He I & 6.0 \\
\hline 4101 & $\mathrm{H} \delta$ & 19.0 \\
\hline 4120 & He I & 2.4 \\
\hline 4144 & $\mathrm{He}$ I & 2.5 \\
\hline 4340 & $\mathrm{H} \gamma$ & 34.0 \\
\hline 4363 & [O III] & 4.0 \\
\hline 4385 & Fe II & 4.5 \\
\hline 4415 & O II & 4.3 \\
\hline 4471 & He I & 5.8 \\
\hline 4489 & O II & 3.2 \\
\hline 4583 & Fe II & 4.5 \\
\hline 4633 & $\mathrm{~N}_{\text {III }}$ & 3.6 \\
\hline 4640 & N III & 6.2 \\
\hline 4647 & C III & 7.8 \\
\hline 4686 & He II & 16.0 \\
\hline 4714 & {$[\mathrm{Ne}$ IV $]$} & 5.0 \\
\hline 4861 & $\mathrm{H} \beta$ & 100.0 \\
\hline 4922 & He I & 9.8 \\
\hline 4959 & [O III $]$ & 5.9 \\
\hline 5007 & [O III $]$ & 21.0 \\
\hline 5015 & He I & 6.7 \\
\hline 5047 & He I & 2.3 \\
\hline 5198 & {$[\mathrm{~N} \mathrm{I}]$} & 3.6 \\
\hline 5233 & {$[\mathrm{Fe}$ VI] } & 2.8 \\
\hline 5316 & Fe II & 4.1 \\
\hline 5876 & He I & 31.7 \\
\hline 6563 & $\mathrm{H} \alpha$ & 632.8 \\
\hline 6678 & He I & 23.3 \\
\hline 7065 & He I & 25.0 \\
\hline 7281 & He I & 6.4 \\
\hline
\end{tabular}

Turnschek et al. 1985). The spectrum of SS73 71 looks similar to the spectrum of Hen 1342 (Medina-Tanco \& Steiner 1995) and AS 289 (Gutiérrez-Moreno et al. 1999), which have spectral types, respectively, of K7-M1 and M 3.5 according to Mürset \& Schmid (1999). We identify the TiO absorption bands in the spectrum of SS7371 at $6200 \AA$, $6852 \AA$ and at $7160 \AA$. The absorption at $6494 \AA$ it is a blend of the absorption features of Ba II, Ca II and Fe I. Around $5890 \AA$ the $\mathrm{NaI}$ is present in absorption. In the range $3100 \AA-5100 \AA$ some absorption features were identified such as Fe I at $3920 \AA, 4045 \AA$ and at $4064 \AA$; Ca II at $3933 \AA$ and the diffuse absorption band at $4430 \AA$.
Table 2. Physical parameters of SS73 71.

\begin{tabular}{lc}
\hline \hline Parameter & Value \\
\hline$V$ & 16.5 \\
$E(B-V)$ & $0.42 \pm 0.06$ \\
$\log N_{\mathrm{e}}^{a} \mathrm{~cm}^{-3}$ & $7.0_{-0.05}^{+0.22}$ \\
$T_{\text {hot }}($ ion $)$ & $35000^{b}$ \\
$T_{\text {hot }}($ ion $)$ & $54000^{c}$ \\
$T_{\text {hot }}($ Ijima $)$ & $110000 \pm 17000 \mathrm{~K}$ \\
He I $\lambda 6678 / 5876$ & $0.6 \pm 0.4$ \\
Infra-red type & $S$ \\
\hline
\end{tabular}

${ }^{a}$ : From [O III] lines; For $T_{\mathrm{e}}=15000 \mathrm{~K}$.

${ }^{b}$ : From $\mathrm{O}^{+2}$ ion.

${ }^{c}$ : From $\mathrm{He}^{+2}$ ion.

The emission spectrum shows the Balmer lines, the major lines of He I, the HeII line at $4686 \AA$ and the oxygen forbidden lines at $4363 \AA, 4959 \AA$ and $5007 \AA$ the latter of which is partly blended with the He I $\lambda 5015$ line in our spectra.

\section{Physical parameters of SS7371}

In this section we will provide some physical parameters of SS7371 such as reddening, electron density, temperature of the hot component and the infra-red type (S-type or D-type). Based on infrared color, symbiotics can be divided into two groups : S- and D-types (Webster \& Allen 1975; Allen 1982). Those that show dust continuum emission between wavelengths 1.0 and $5.0 \mu \mathrm{m}$ are classified as D-type, and these systems contain a mass-losing Mira variable as a cool component. Those with a normal stellar spectrum in the infrared are classified as S-types. Table 2 contains the results.

Reddening estimates were based on Netzer's (1975) curves for $\mathrm{H} \beta / \mathrm{H} \gamma$ versus $\mathrm{H} \alpha / \mathrm{H} \beta$. It was assumed that the reddening law can be represented by the standard interstellar extinction curve $f(\lambda)$ (Seaton 1979).

It was not possible to make good plama diagnostics with the emission lines available. In fact we do not have lines for electron temperature determination for densities as high as those involved in these kinds of symbiotic systems. The [O III] lines at $5007 \AA, 4959 \AA$ and $4363 \AA$ are not good indicators of temperature but are approriate for electron densities. Using the [O III] line ratios corrected for reddening and assuming $T_{\mathrm{e}}=15000 \mathrm{~K}$ we obtained the electron density.

The temperature of the hot component was determined using the method proposed by Mürset \& Nussbaumer (1994) ( $\left.T_{\text {ion }}\right)$ (hereafter MN94) and Ijima (1981) $\left(T_{\mathrm{Ijima}}\right)$. The MN94 method has the advantage that it takes into considaration the observed ion with the highest ionization potential, but may fail for the S-type systems, because some forbidden lines may be absent because of the high density. In the method of Ijima (1981) the temperature derived is an upper limit for the hot component 
since optical depths may alter the intensity of Balmer lines.

Proga et al. (1994) demonstrated that He I emission line ratio can be used to distinguish between S-type (stellar continuum) and D-type (dust) symbiotic stars, besides their IR-colors. Since the S-type systems have higher electron densities $\left(N_{\mathrm{e}} \approx 10^{9} \mathrm{~cm}^{3}\right)$ than the D-type systems $\left(N_{\mathrm{e}} \approx 10^{6} \mathrm{~cm}^{3}\right)$, the emission-line ratio, as defined by the ratio $I(6678 / 5876)$, distinguishes the two types. Those that have $I(6678) / I(5876) \approx 0.25$ are $\mathrm{D}$ type and those that have $I(6678) / I(5876) \geq 0.50$ are S-types.

\section{Summary and conclusions}

Based on a spectroscopic survey between $3200 \AA-5200 \AA$ and $3500 \AA-8000 \AA$ of $\mathrm{H} \alpha$ emission-line stars from the SS73 sample we discovered a new symbiotic star (SS73 71). Its continuum spectrum seems to indicate the spectral type of late K-early M with Balmer lines, helium lines and forbidden lines of oxygen in emission. We also provide a table with the identified features and some physical parameters.

\section{References}

Allen, D. A. 1978, MNRAS, 184, 601

Allen, D. A. 1982, in The Nature of Symbiotic Stars, ed. M. Friedjung, \& R. Viotti (Dordrecht: Reidel), IAU Colloq., 70,27
Belczyński, K., Mikolajewska, J., Munari, U., Ivison, R. J., \& Friedjung, M. 2000, A\&AS, 146, 407

Gutiérrez-Moreno, A., Moreno, H., \& Costa, E. 1999, PASP, 111,571

Hamuy, M., Suntzeff, N. B., Heathcote, S. R., et al. 1994, PASP, 106, 566

Ijima, T. 1981, in Photometric and Spectroscopic Binary Systems, ed. E. B. Carling, \& Z. Kopal (Dordrecht, Reidel), 517

Jacoby, G. H., Hunter, D. A., \& Christian, C. A. 1984, ApJSS, 56,257

Landaberry, S. J. C., Pereira, C. B., \& de Araújo, F. X. 2001, A\&A, 376, 917

Medina-Tanco, G., \& Steiner, J. E. 1995, AJ, 109, 1770

Mürset, U., \& Nussbaumer, H. 1994, A\&A, 282, 586 (MN94)

Mürset, U., \& Schmid, H. M. 1999, A\&AS, 137, 473

Netzer, H. 1975, MNRAS, 171, 395

Oke, J. B. 1974, ApJS, 27, 21

Pereira, C. B., Landaberry, S. J. C., \& Conceição, F. 1998, AJ, 116, 1971

Proga, D., Mikolajewska, J., \& Kenyon, S. J. 1994, MNRAS, 268,213

Sanduleak, N., \& Stephenson, C. B. 1973, ApJ, 185, 899 (SS73)

Seaton, M. J. 1979, MNRAS, 187, 73

Turnshek, D. E., Turnshek, D. A., Craine, E. R., \& Boeshaar, P. C. 1985, An Atlas of Digital Spectra of Cool Stars (Western Research Co. Tucson, Arizona)

Webster, B. L., \& Allen, D. A. 1975, MNRAS, 171, 171 